\title{
Malaria Outbreak Investigation in Pastoral Communities of BenaTsemay District, Southern Ethiopia: A Case Control Study
}

\author{
Mebratu Shite Wondimu ${ }^{1, *}$, Endrias Markos Woldesemayat ${ }^{1}$, Tsehay Ayele Aselle ${ }^{2}$ \\ ${ }^{1}$ School of Public Health, College of Medicine and Health Science, Hawassa University, Hawassa, Ethiopia \\ ${ }^{2}$ School of Public Health, College of Health Sciences, Addis Ababa University, Addis Ababa, Ethiopia
}

Email address:

mebratushite16@gmail.com (M. S. Wondimu), Endromark@gmail.com (E. M. Woldesemayat),

Shayeayele@yahoo.com (T. A. Aselle)

${ }^{*}$ Corresponding author

\section{To cite this article:}

Mebratu Shite Wondimu, Endrias Markos Woldesemayat, Tsehay Ayele Aselle. Malaria Outbreak Investigation in Pastoral Communities of BenaTsemay District, Southern Ethiopia: A Case Control Study. American Journal of Health Research. Vol. 7, No. 3, 2019 , pp. $31-37$.

doi: 10.11648/j.ajhr.20190703.11

Received: March 4, 2018; Accepted: July 10, 2019; Published: July 26, 2019

\begin{abstract}
Over 60\% (60 million) of Ethiopian populations live in areas at risk for malaria. On June $17 / 2017$, surveillance data from BenaTsemay district showed that the district was experiencing an unusual high number of malaria cases in three kebeles (lowest administrative unit). We investigated the outbreak to describe malaria epidemiology in the district, identify population risk factors, and guide intervention measures for the community. Malaria case records from the District Health Office were reviewed to describe the outbreak. Unmatched case-control study was conducted with 60 randomly selected cases and 119 controls using a semi-structured questionnaire. Malaria cases were confirmed by either microscopy or malaria rapid diagnostic tests (RDTs) from June 5 to July 23/2017. Controls were persons with no diagnosis of malaria, and lives in similar kebele. Environmental assessment, observation of ownership and utilization of insecticide treated bed-nets (ITNs) were carried out. Multivariable regression model analysis was conducted to identify independent malaria risk factors. There were 648 malaria cases (50.3\%-males) from three kebeles with no deaths recorded. The overall attack rate (AR) was $114 / 1000$ populations, and it was highest in Sille kebele (167/1000 populations) and among 5-14 years old (179/1000 populations). Age $<5$ years ( $\mathrm{AOR}=8.5 ; 95 \% \mathrm{CI}: 1.2-59)$, living near mosquito breeding sites (AOR=6.5; 95\%CI: 1.15-36.5), irregular use of ITNs (AOR=8.7; 95\%CI: $1.5-49.6$ ), inadequate ITNs per family size (AOR=13.4; 95\%CI: $1.9-93)$ predicted having of malaria. Wearing long sleeved clothes was a protective factor (AOR=0.6; 95\%C.I: 0.004-0.96). Several unmanaged stagnated water sources with Anopheles mosquito larvae, and the use of ITNs for other purposes were observed. The outbreak was associated with presence of nearby vector-breeding sites, and poor access and utilization of ITNs. Adequate information should be given along ITNs for the community in addition to early management of nearby vectorbreeding sites to prevent similar outbreak in future.
\end{abstract}

Keywords: Malaria, Outbreak, Bena Tsemay District, Pastoral, Sothern Ethiopia

\section{Introduction}

According to the World Health Organization (WHO), an estimated 3.3 billion people are at risk of malaria, of whom 1.2 billion are at high risk [1]. In 2015, there were about 212 million new cases of malaria and 429,000 deaths of malaria worldwide. The WHO African Region accounted for $90 \%$ and $92 \%$ global malaria cases and deaths respectively and children aged under five years account for $70 \%$ of all malaria deaths [2].

An estimated 60\% (60 million people) of Ethiopian populations live in areas at risk for malaria. Malaria is mainly seasonal with unstable transmission in the highland fringe areas and of relatively longer transmission duration in lowland areas [3]. According to report of Ethiopian Ministry of Health, in 2014, there were about 2,383,010 malaria illnesses and 450 
malaria deaths in the country [4]. This is excluding epidemics when the rates of morbidity and mortality increase dramatically (i.e. 3-5 fold) the normal rate [3].

In Ethiopia, major epidemic in between July and December of 1958 affected three quarters of the densely populated areas of central highlands causing over 3.5 million cases of malaria and an estimated 150,000 deaths [5]. The disease has also economic burden in the country [6] as malaria epidemics usually coincide with farming seasons, thus reducing labor productivity and in effect of jeopardizing the household economy [7].

Benna Tsemay district is one of moderate malaria transmission areas in Southern Ethiopia. The prevalence of malaria in the district, in 2012, was $6.1 \%$ with predominant species of plasmodium falciparum (64.3\%) followed by plasmodium vivax (21.4\%) [8]. On June 17 2017, surveillance data on malaria obtained from the district health office showed that the district was experiencing an unusual high number of malaria disease. It reported 182 malaria cases in WHO epidemiologic week 25 of 2017 compared with only 48 cases in 2016 in the same epidemiologic week. In response, on June 24/2017, rapid response team was deployed to the affected district to verify the existence of the outbreak, identify risk factors, and assist in intervention measures.

\section{Methods}

\subsection{Study Area, Period, and Populations}

Benna Tsemay district is one of the eight districts in South Omo Zone, southern Ethiopia. Located at $745 \mathrm{~km}$ Southwest of Addis Ababa, it has an estimated total population of 68,881 . Livelihoods are largely based on rearing animals (pastorals) [9]. The district is divided into 32 kebeles. The altitude of the district is $1,500 \mathrm{~m}$ above sea level with annual temperatures ranging from $26^{\circ} \mathrm{C}$ to $40^{\circ} \mathrm{C}$. The average annual rainfall is $800 \mathrm{~mm}$. The district has a long dry season from December to the beginning of March while June and July is a short dry season. Six Health Centers and 32 Health Posts are found in the district. The investigation was conducted from June 5 to July 23/2017.

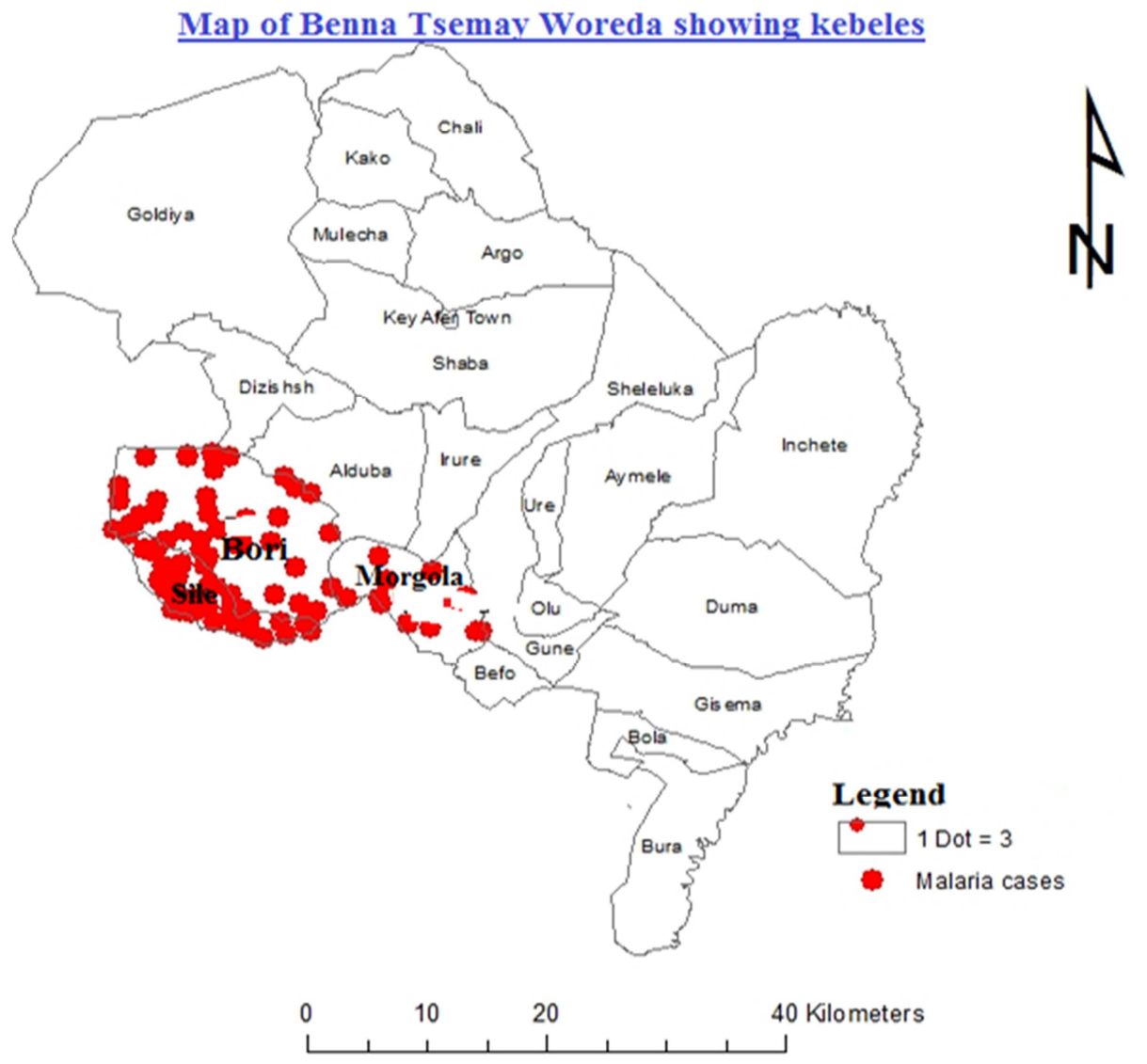

Figure 1. Dot map showing kebeles that were affected by malaria outbreak, Benna Tsemay District, Southern Ethiopia, July 2017.

\subsection{Operational Definitions}

Threshold: Critical number of malaria cases that is used to confirm the emergence of malaria epidemic so as to step-up appropriate control measures

Near mosquito breeding site: Living at about $1 \mathrm{~km}$ radius of stagnated water
Adequate ITNs: At least one ITN per two individuals per household.

\subsection{Laboratory Methods}

Malaria cases were confirmed by microscopy at Health Center level and by malaria rapid diagnostic tests (RDTs) at 


\section{Health Post levels.}

\subsection{Descriptive Study}

Malaria case was defined as any person with fever with plasmodium parasites confirmed by either microscope or RDT in residents of Benna Tsemay district from June 5 to July 23/2017.

Malaria records obtained from the District Health office were reviewed. The record contained information on variables such as name, location, age, and sex of the patient, date of onset of the illness, date of health facility visit, symptoms, laboratory investigation and its result, patient status (inpatient/outpatient), and treatment outcome (live/dead). Reviewed data were disaggregated into kebeles. The disaggregation showed that about $160(90 \%)$ of the cases had been reported from 3 out of 32 kebeles namely Sille, Bori and Morgola (all from Alduba cluster). The threshold (a critical number of malaria cases that is used to confirm the emergence of malaria epidemic) was identified to define the epidemic in terms of the previous experience of the disease.

\subsection{Analytical Epidemiology}

The dependent variable for the study was malaria illness while independent variable includes variables such as age, sex, occupation, ethnicity, religion, marital status, educational status, sleeping area, staying outside overnight, availability of ITNs in the households, number of ITN in the household, utilization of ITN, presence of breeding site near home, type of breeding site and knowledge regarding malaria disease. Knowledge about the disease, mode of transmission and prevention method was measured by comparing mean score responses to 10 items. Those with scores at or below the mean were classified as having "poor knowledge" while those with scores above the mean were categorized as having "good knowledge".

Unmatched case-control study was conducted with 60 randomly selected cases and 119 community controls using face-to-face a structured interview administered questionnaire. Cases were person with diagnosis of malaria in the study communities. Controls were persons with no history of fever and diagnosis of malaria, live in similar kebele and neighborhood to cases. Data were entered into Epi-Info 7 and analyzed using Statistical Package for Social Science version 20 (SPSS-20). Variables with p-value $<0.25$ in bivariate analysis were entered in multiple logistic regression analysis to examine the effect of an independent variables on the outcome variable. The association between dependent and independent variables was determined using odds ratio of $95 \%$ CI at $\mathrm{p} \leq 0.05$.

\subsection{Environmental Assessment}

Environmental assessment for availability of stagnant water, uncovered plastic water containers, broken glass bottles, and other potential mosquito breeding sites was carried out. Moreover, observation on possession and utilization of ITNs was employed in randomly selected 45 households in the affected kebeles.

\section{Results}

\subsection{Existence of Malaria Outbreak}

As we could not obtain five-year weekly malaria data, the existence outbreak was confirmed by doubling last year's weekly data as a threshold based on the national malaria guideline [3]. The alert threshold had been crossed in $24^{\text {th }}$ WHO epidemiologic week (Figure 2.)

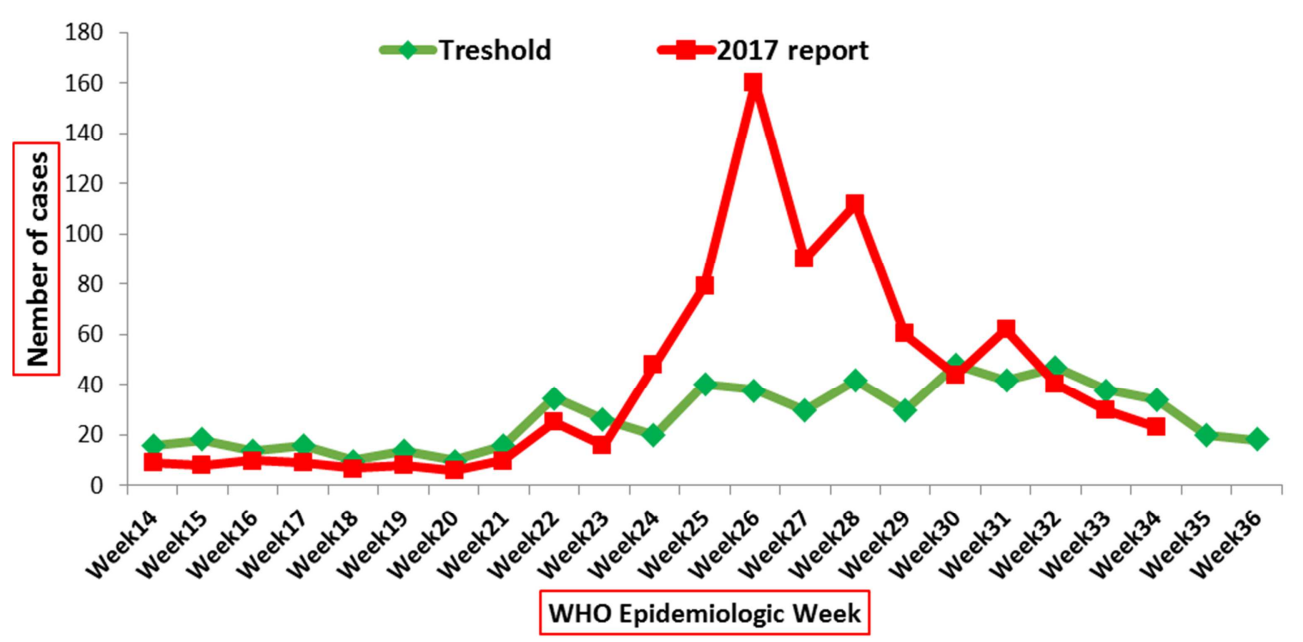

Figure 2. Trend of malaria cases by WHO epidemiologic week in Alduba cluster of Benna Tsemay District, Southern Ethiopia, July 2017.

\subsection{Descriptive Epidemiology}

Of the 1022 malaria blood tests done, 648 of them were positive for malaria with positivity rate of $63.4 \%$ (range, 49.4\%-80\%). The proportion of Plasmodium falciparum was
$602(92.9 \%)$ with no mixed species. Half (50.3\%) of the cases were males. The median (IQR) age of the study participants was $24.5(20-38)$ years. The admission rate was $15 \%$ with no death recorded. A little bit higher than half, 336 
$(51.9 \%)$ of the cases were reported from Bori kebele. The overall AR was 114 per 1000 populations. The descriptive epidemiology of the disease is summarized in Table 1 as follows.

Table 1. Description of malaria cases by age, sex, residence, and attack rate, Benna Tsemay District, Southern Ethiopia, July 2017.

\begin{tabular}{|c|c|c|c|c|}
\hline \multicolumn{2}{|c|}{ Description } & \multirow{2}{*}{$\begin{array}{l}\text { Total population (risk) } \\
821\end{array}$} & \multirow{2}{*}{$\begin{array}{l}\text { Number of malaria cases } \\
85\end{array}$} & \multirow{2}{*}{$\begin{array}{l}\text { AR per } 1000 \text { populations } \\
115.7\end{array}$} \\
\hline & $<5$ & & & \\
\hline Age & $5-14$ & 1350 & 248 & 176.3 \\
\hline & $>15+$ & 3511 & 315 & 89.7 \\
\hline \multirow{2}{*}{ Sex } & Male & 2830 & 326 & 115 \\
\hline & Female & 2852 & 322 & 112 \\
\hline \multirow[b]{2}{*}{ Kebele } & Bori & 2201 & 336 & 152.6 \\
\hline & Sille & 1178 & 197 & 167.2 \\
\hline Total & & 5683 & 648 & 114 \\
\hline
\end{tabular}

As illustrated from the Epic curve below (Figure 2.), the pattern of the outbreak is propagative type.

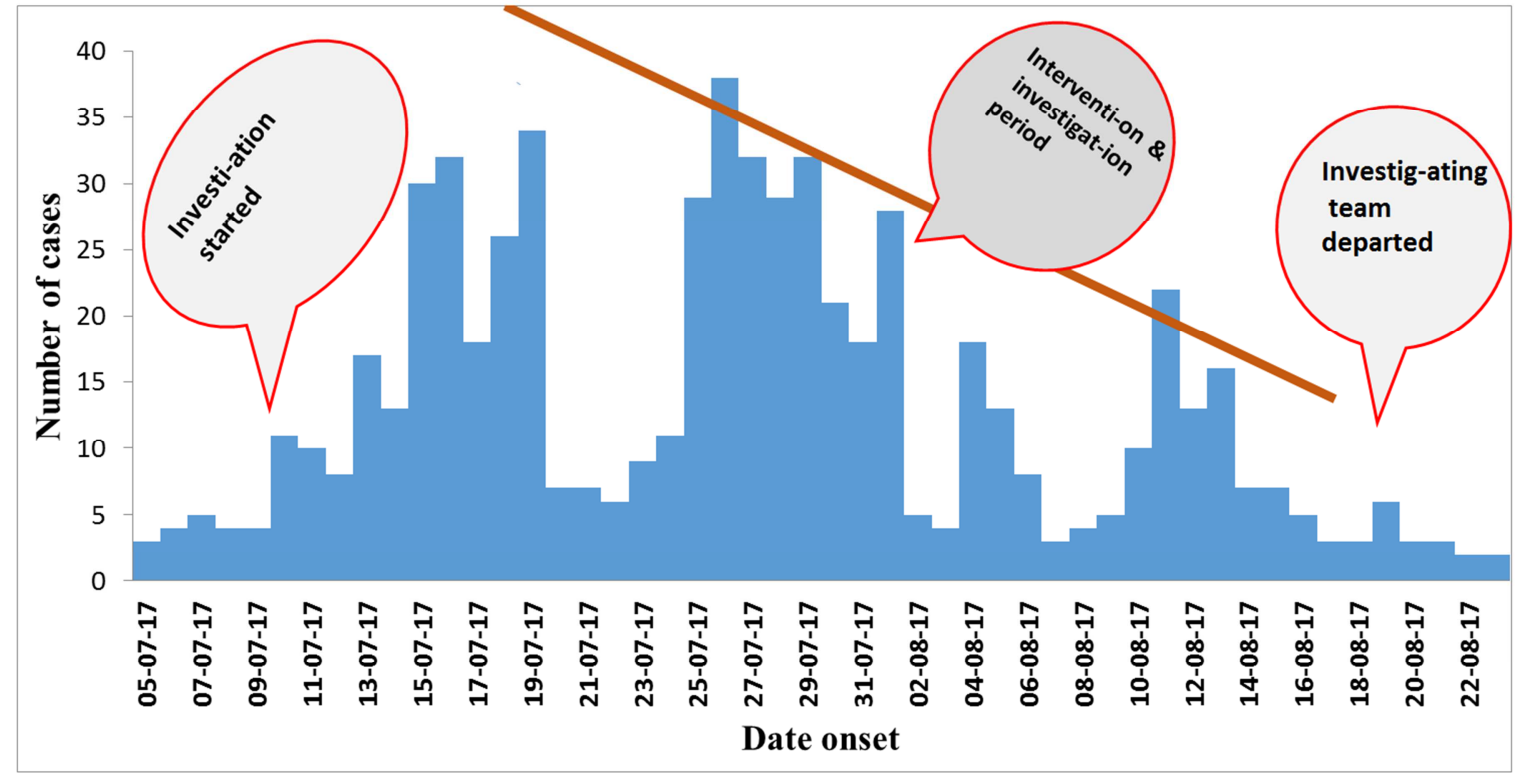

Figure 3. Distribution of malaria cases by date of onset in Benna Tsemay District, Southern Ethiopia, July 2017.

\subsection{Analytical Epidemiology}

\subsubsection{Socio-Demographic Description of the Respondents}

During the study period, 178 eligible study participants were selected and interviewed making response rate of 99. Male participants were 33 (56\%) among cases and 68 (57\%) among controls (Table 2).

Table 2. Socio-demographic description of the study participants Benna Tsemay District, Sothern Ethiopia, July 2017.

\begin{tabular}{|c|c|c|c|c|}
\hline \multirow{2}{*}{ Variable Description } & & Case $(n=59)$ & Control $(n=119)$ & Total $(n=178)$ \\
\hline & & $\mathbf{N}(\%)$ & $\mathbf{N}(\%)$ & $\mathbf{N}(\%)$ \\
\hline \multirow{3}{*}{ Age } & $<5$ & $17(29 \%)$ & $5(4 \%)$ & $22(12 \%)$ \\
\hline & $5-14$ & $6(10 \%)$ & $12(10 \%)$ & $18(10 \%)$ \\
\hline & $>15+$ & $36(61 \%)$ & $102(86 \%)$ & $138(78 \%)$ \\
\hline \multirow{2}{*}{ Sex } & Male & $33(56 \%)$ & $68(57 \%)$ & $101(57 \%)$ \\
\hline & Female & $26(44 \%)$ & $51(43 \%)$ & $77(43 \%)$ \\
\hline \multirow{3}{*}{ Marital Status } & Married & $46(78 \%)$ & $90(76 \%)$ & $136(76 \%)$ \\
\hline & Single & $10(17 \%)$ & $22(18.4 \%)$ & $32(18 \%)$ \\
\hline & Others & $3(5 \%)$ & $7(5.6 \%)$ & $14(6 \%)$ \\
\hline \multirow{3}{*}{ Educational Status } & Illiterate & $42(71.2 \%)$ & $91(76.4 \%)$ & $133(74.7 \%)$ \\
\hline & primary & $12(20.3 \%)$ & $19(16 \%)$ & $31(17.4 \%)$ \\
\hline & Secondary and above & $5(8.5 \%)$ & $9(7.6)$ & $14(7.9 \%)$ \\
\hline \multirow{4}{*}{ Occupation } & Pastoralist & $16(27 \%)$ & $61(51 \%)$ & $77(43 \%)$ \\
\hline & Housewife & $33(56 \%)$ & $33(28 \%)$ & $66(37 \%)$ \\
\hline & Student & $7(12 \%)$ & $19(26 \%)$ & $26(15 \%)$ \\
\hline & Employed & $3(5 \%)$ & $6(5 \%)$ & $3(2 \%)$ \\
\hline
\end{tabular}


The mean (SD) age for cases and controls were 19.46 (15.6) and 29.3 (15.7) years respectively. About $46(78 \%)$ cases and $90(76 \%)$ of controls were married. Forty-two $(71.2 \%)$ of cases and ninety-one $(76.4 \%)$ of controls were illiterate.

\subsubsection{Risk Factors Associated with the Outbreak}

Children less than five years old were about nine times more likely to develop malaria ( $\mathrm{AOR}=8.5 ; 95 \% \mathrm{CI}: 1.2-59)$ as compared to people $>15$ years old. Those who live nearby mosquito breeding site (at a distance of $\sim 1 \mathrm{Km}$ ) were nearly seven times more likely to develop malaria (AOR: 6.5; 95\%CI: 1.15- 36.5).

Table 3. Bivariate and Multivariate analysis of risk factors for Malaria outbreak in Benna Tsemay District, Sothern Ethiopia, July 2017.

\begin{tabular}{|c|c|c|c|c|c|}
\hline \multirow{2}{*}{ Variables } & \multirow{2}{*}{ Variable coding } & \multicolumn{2}{|c|}{ Status } & \multirow{2}{*}{$\operatorname{COR}(95 \%$ CI $)$} & \multirow{2}{*}{$\operatorname{AOR}(95 \%$ CI) } \\
\hline & & Case & Control & & \\
\hline \multirow{3}{*}{ Age of the respondent } & $<5$ years & 21 & 9 & $7.7(3.2-18.7)$ & $8.5(1.2-59.0)^{*}$ \\
\hline & $5-14$ years & 9 & 14 & $2.12(0.8-5.5)$ & $1.31(0.11-15.8)$ \\
\hline & $\geq 15$ & 29 & 96 & 1 & 1 \\
\hline \multirow{2}{*}{ Presence of mosquito breeding near home } & Yes & 48 & 26 & \multirow{2}{*}{$15.6(7.01-34.3)$} & \multirow{2}{*}{$6.5(1.2-36.5)^{*}$} \\
\hline & No & 11 & 93 & & \\
\hline \multirow{2}{*}{ Frequency of ITN use } & Sometimes & 14 & 19 & $4.34(2.23-8.42)$ & $8.7(1.5-49.6)$ \\
\hline & Always & 4 & 40 & 1 & 1 \\
\hline \multirow{2}{*}{ Wearing protective clothes } & Yes & 10 & 48 & $0.3(0.14-0.65)$ & $0.6(0.004-0.9)^{*}$ \\
\hline & No & 49 & 71 & 1 & 1 \\
\hline \multirow{2}{*}{ Number of ITN per household } & Inadequate & 10 & 12 & $4.9(1.58-15)$ & $13.4(1.9-93)^{*}$ \\
\hline & Adequate & 7 & 47 & 1 & 1 \\
\hline \multirow{2}{*}{ Knowledge on malaria transmission and prevention } & poor & 35 & 33 & $3.8(1.97-7.3)$ & $0.83(0.12-5.8)$ \\
\hline & Good & 24 & 86 & 1 & 1 \\
\hline
\end{tabular}

NB: *- Statistically significant at $\mathrm{p}<0.05$.

Those respondents who did not use ITN regularly were about nine times higher risk of developing malaria (AOR; 8.7; 95\%CI: 1.5-49.6). The odds of contracting malaria among respondents who lived in households with inadequate number of ITNs per family size was about 13 times (AOR: 13.4: 95\%CI: 1.9-93). On another hand, respondents who wore long sleeved clothes were less likely to develop malaria disease ( $\mathrm{AOR}=0.6 ; 95 \% \mathrm{CI}$ : 0.004-0.96; $\mathrm{P}=0.047$ ) compared to their counterpart. Having good knowledge related to malaria prevention and transmission had no association with malaria illness in multivariate analysis $(\mathrm{AOR}=0.83 ; 95 \% \mathrm{CI}$; $0.12-5)$.

\subsubsection{Environmental Assessment}

In all assessed kebeles, environmental investigation was conducted. Larvae of mosquitoes in stagnant water were observed by naked eye. Of the visited 45 households, more than half $24(53.3 \%)$ had no any ITN. A little bit higher than an half, $23(51.1 \%)$ of the respondents who owned ITN did not use it regularly. There were worn out/old ineffective ITNs in nine $(20 \%)$ households. Another $9(20 \%)$ of visited households were using ITNs for other purposes such as making rope, as covering for grain and hay, curtain. Indoor residual spray (IRS) had not been applied since 2015 in each visited households. Another potential mosquito breeding sites like uncovered plastic water containers, broken glass bottles were not observed in the visited kebeles.

\section{Discussion}

This study confirmed the existence of malaria outbreak in three kebeles. Factors like age, poor ITNs access, and utilization, and presence of nearby stagnated water contributed for the existence of the epidemic.

About 602 (92.9\%) of the confirmed malaria cases were due to plasmodium falciparum ( $p$. falciparum) species. This finding is higher than the usual proportion malaria species in Ethiopia whereas about $60 \%$ of the species is $p . f(4)$. This finding also disagrees with previous study conducted in the same area to assess prevalence and risk factors of malaria (2012) where proportion of $p$. falciparum accounted 64.3\% [8]. During malaria epidemics, the dominance of $p$. falciparum over the other species is common in Ethiopia [10] and unlike this study; the previous study was conducted during malaria stable transmission period. The overall AR is three times higher than a study conducted in Northern Ethiopia [11]. Higher AR might be due to difference in the study populations and area.

The AR was highest in Sille kebele (167.2 per 1000 populations) and this might be due to presence of multiple mosquito breeding nearby residents of Sille Kebele compared to the other two kebeles-9 (56.3\%) of 16 mosquito breeding sites were in this kebele. Age specific AR was highest among children 5-14 years (176.3/1000 populations) and this finding is in line with study from Ankasha District, North Ethiopia [12].

In another study [13], males are highly affected by malaria than females, which is perceived to be the fact that males are usually engaged in outdoor activities. In contrast, the distribution is nearly equal in our study. This could be related to nature of the community: first, women are also involved in outdoor activities particularly walking some kilometers to fetch water between dusk and dawn. Second, being engaged in indoor activities does not decrease risk of contracting malaria as the nature of the home found in the community has not been screened by itself.

Being $<5$ years were about nine times more likely to 
develop malaria disease $(\mathrm{AOR}=8.5 ; 95 \% \mathrm{CI}: 1.2-59)$ compared to age 15 years and above. This finding agrees with study conducted in Butajira, Ethiopia [14]. Immunity to malaria develops slowly after several infections and children need at least five years to develop their immunity [15]. Those people living at about $1 \mathrm{~km}$ radius of mosquito breeding site were nearly seven times as likely to develop malaria (AOR: 6.5; 95\%CI: $1.15-36.5$ ) compared to those living mosquito free breeding site. This finding is in line with study conducted in Gamo Gofa zone, Southern Ethiopia [16]. Multiple muddy rainwater collection and water pools creating stagnated water can support mosquito breeding unless polluted. Environmental observation also supported the presence multiple fresh water collections created after rainy season. The odds of developing malaria among people who used ITN occasionally was nearly nine times (AOR; 8.7; 95\%CI: 1.5-49.6) compared to who use ITNs frequently. Similar finding was also reported from study in Ethiopia [11] and India [17]. Respondents who lived in households with inadequate number of ITNs were 13 times more likely to develop malaria disease (AOR: 13.4: 95\%CI: 1.9-93). Inadequate ITN in households might enforce the community to utilize the ITNs infrequently. The participants who wore long sleeved clothes were about $96 \%$ less likely to develop malaria disease (AOR $=0.041 ; 95 \% \mathrm{CI}: 0.002-0.871)$ than their counterparts. Clothing can offer protection from biting insects when it is of a thickness and texture through which insects cannot easily bite [18].

Our finding did not reveal any supporting evidence that sleeping area and housing status to show a statistically significant difference between cases and controls. These findings could be related to difference social and demographic factors that might not be accounted in. i.e., most of the housing nature found in this community is not screed so that sleeping in or out of the house has no protective value from mosquito biting.

The national malaria prevention and control strategy recommends application of IRS as at least once in a year with $100 \%$ coverage, and at least one ITN per two people in malaria high-risk areas [4, 19]. Despite this fact, IRS was not applied prior to the outbreak and early replacement of ITNs was not done. This might be related to poor planning and /or implementation of malaria prevention and control strategies. Households who had been using the ITNs other than its intended purpose were also observed. This could be due to poor monitoring of the communities after distributing the ITNs.

\section{Public Health Intervention}

During the investigation period, active case search and case management was carried out based on national malaria treatment guideline. More than 2,500 worn out/ineffective ITNs for 830 households were replaced. Both opening of temporally stagnated water through community participation and application of larvicide (abet chemical) on the breeding site was carried out. Focal spray of IRS (with propoxurs) for 2364 unit structure was conducted. Health education was given to the communities in order to enhance their awareness on the cause, transmission, and prevention of the disease.

\section{Conclusion}

Generally, the outbreak is related poor application of malaria prevention and control strategies. Being $<5$ years old, presence of nearby vector-breeding sites, poor access and utilization of ITNs were significantly associated risk factors for the occurrence of the outbreak.

\section{Recommendation}

To prevent subsequent similar malaria outbreaks in the district, the following action points have been recommended. The local health officials should apply all malaria prevention and control strategies on early. Since the pastoral way of life and the climate have a huge impact on the lifespan and sustainability of the mosquito nets, early replacement of worn out ITNs is vital. Moreover, the communities should refrain from using the ITNs other than its intended use.

\section{Abbreviations}

AOR: Adjusted Odds Ratio, AR: attack rate, COR: Crude Odds Ratio, IRS: Indoor residual spraying, ITNs: Insecticide treated bed-nets, RDT: Rapid diagnostic tests, WHO: World Health Organization

\section{Authors Contributions}

MS designed the study, supervised data collection, performed data analysis, and interpretation, and drafted the manuscript. EM and TA assisted in the design, data analysis, and interpretation, and reviewed the manuscript. All authors read and approved the final manuscript.

\section{Acknowledgements}

The authors would like to thank Ethiopian Public Health Institute for the financial support.

\section{Competing Interest}

The authors declare that they have no competing interests.

\section{Availability of Data and Materials}

The datasets used and analyzed in the study are available from the corresponding author up on reasonable request.

\section{References}

[1] World Health Organization (2014): World Malaria Report. [cited on July 15, 2017]. Available from: http://www.who.int/malaria/publications/world_malaria_repor t_2014/en/. 
[2] World Health Organization (2016): World Malaria Report [Internet], cited on July 15, 2017]. Available from: http://www.who.int/malaria/publications/world-malariareport-2016/report/en/.

[3] Ethiopian Federal Ministry of Health (2016): Malaria Diagnosis and Treatment Guidelines for Health Workers in Ethiopia, Addis Ababa, Ethiopia.

[4] Ethiopian Federal Ministry of Health (2014). National Malaria Strategic Plan (2014-2020);. Addis Ababa 2014.

[5] Ethiopian Federal Ministry of Health (1999): Guidelines for Malaria Epidemic Prevention and Control in Ethiopia,. Addis Ababa, Ethiopia.

[6] Deressa W. et al.: Economic cost of epidemic malariato house holds in rural Ethiopia. Trop Med Int Health 2007; 12 (10): 1148-56.

[7] Anthony E. Kiszewski T: A review of the clinical and epidemiological burds of epidemic malaria. American Society of Tropical Medicine and Hygiene. 2004; 71 (2): 128-35.

[8] Debo W, Kassa H (2016): Prevalence of malaria and associated factors in Benna Tsemay district of pastoralist community, Southern Ethiopia.

[9] Annual Review Meeting Report of South Omo Zone Health Department (2016), Jinka, Ethiopia.

[10] Yeshiwondim A. et. al,: Spatial analysis of malaria incidence at the village level in areas with unstable transmission in Ethiopia. International Journal of Health Geographics. 2009; 8 (1): 5 .

[11] Gemechu B. et. al.: A Malaria Outbreak in Ameya Woreda,
South-West Shoa, Oromia, Ethiopia, 2012: Weaknesses in Disease Control, Important Risk Factors. American Journal of Health Research. 2015; 3 (3): 125-9.

[12] Worku M: Epidemiological Analysis of Malaria Outbreak in Ankesha District, Awi Zone, Amhara Region, Ethiopia, 2012: Weaknesses in Control Measures and Risk Factors. Science Journal of Public Health. 2016; 4 (2): 132.

[13] Graves M. et. al.: Factors associated with mosquito net use by individuals in households owning nets in Ethiopia. Malaria Journal 2011 10: 354. 2011; 10: 354.

[14] Woyessa A. Malaria risk factors in Butajira area, south-central Ethiopia: a multilevel analysis. Malaria Journal 2013; 12: 273.

[15] Center for Disease Prevention and Control (2017): Factors Affecting Malaria Transmission: Accessed on July, 2017, available from: http://www.open.edu/openlearncreate/mod/oucontent/view.ph $\mathrm{p} ? \mathrm{id}=89 \&$ printable $=1$.

[16] Tarekegn N (2014): Malaria Outbreak Investigation, ChanoMile kebele, Arbaminch Zuria Woreda, Gamogofa Zone.

[17] Yadav K: Socio-economic determinants for malaria transmission risk in an endemic primary health centre in Assam, India; Infectious Diseases of Poverty. 2014; 3: 19.

[18] World Health Organization (1997); Vector Control Methods: Available at: http://www.who.int/water_sanitation_health/resources/vector0 59to87.pdf.

[19] USAID; President's Malaria Initiative Ethiopia Malaria Operation Plan (2017), Addis Ababa Ethiopia. 